(C) 2021, The Authors. Published by Elsevier Inc. and Fass Inc. on behalf of the American Dairy Science Association ${ }^{\circledR}$. This is an open access article under the CC BY-NC-ND license (http://creativecommons.org/licenses/by-nc-nd/4.0/).

\title{
Genotyping strategies for maximizing genomic information in evaluations of the Latxa dairy sheep breed
}

\author{
I. Granado-Tajada, ${ }^{1 *}$ (다 L. Varona, ${ }^{2}$ and E. Ugarte ${ }^{1}$ () \\ ${ }^{1}$ Department of Animal Production, NEIKER-BRTA Basque Institute of Agricultural Research and Development, Agrifood Campus of Arkaute s/n, \\ E-01080 Arkaute, Spain \\ ${ }^{2}$ Departamento de Anatomía Embriología y Genética Animal, Instituto Agroalimentario de Aragón (IA2), Universidad de Zaragoza, \\ 50013 Zaragoza, Spain
}

\section{ABSTRACT}

Genomic selection has been implemented over the years in several livestock species, due to the achievable higher genetic progress. The use of genomic information in evaluations provides better prediction accuracy than do pedigree-based evaluations, and the makeup of the genotyped population is a decisive point. The aim of this work is to compare the effect of different genotyping strategies (number and type of animals) on the prediction accuracy for dairy sheep Latxa breeds. A simulation study was designed based on the real data structure of each population, and the phenotypic and genotypic data obtained were used in genetic (BLUP) and genomic (single-step genomic BLUP) evaluations of different genotyping strategies. The genotyping of males was beneficial when they were genetically connected individuals and if they had daughters with phenotypic records. Genotyping females with their own lactation records increased prediction accuracy, and the connection level has less relevance. The differences in genotyping females were independent of their estimated breeding value. The combined genotyping of males and females provided intermediate accuracy results regardless of the female selection strategy. Therefore, assuming that genotyping rams is interesting, the incorporation of genotyped females would be beneficial and worthwhile. The benefits of genotyping individuals from various generations were highlighted, although it was also possible to gain prediction accuracy when historic individuals were not considered. Greater genotyped population sizes resulted in more accuracy, even if the increase seems to reach a plateau.

Key words: genomic selection, genotyping strategy, Latxa breed, prediction accuracy

Received November 30, 2020.

Accepted February 12, 2021.

*Corresponding author: igranado@neiker.eus

\section{INTRODUCTION}

The procedures of genetic evaluation of livestock animals have been revolutionized over the last 2 decades by the introduction of the genomic selection (GS; Meuwissen et al., 2001). Its implementation has been shown to be beneficial in simulations (Meuwissen et al., 2001), as well as in several livestock species (VanRaden et al., 2009; Ibañez-Escriche and Simianer, 2016; VanRaden, 2020). There is consensus on the benefits of GS for shortening the generation interval and increasing prediction accuracies of genetic values, especially in those individuals without their own phenotypic data. Therefore, its implementation makes it possible to achieve higher genetic gains compared with classical selection (Ibañez-Escriche and Gonzalez-Recio, 2011).

The reliability of genomic prediction is influenced by several factors, including the specific features of the trait of interest (heritability, genetic architecture, number and distribution of genes, and linkage disequilibrium between prediction markers and QTL), the characteristics of the population, and the design of the genotyped reference population (size, sex ratio, or the relationship of genotyped individuals within them, with candidates for selection, and with the overall population; Lund et al., 2016; Schöpke and Swalve, 2016; van den Berg et al., 2019).

There is a general agreement that before the application of GS to a breeding program, it is important to have adapted it to the biological, productive, and economic circumstances of the population (Boichard et al., 2016). In small ruminant populations, as is the case of Latxa breeds, the potential gain in accuracy provided by molecular information may be lower than in other species because of the low linkage disequilibrium (Kijas et al., 2014), due to higher effective population size and introgression of other populations (Ibañez-Escriche and Gonzalez-Recio, 2011; Rupp et al., 2016). In addition, the population size is usually small, they have a short generation interval, the use of AI is limited, and there 
is a considerable percentage of unknown parents in the pedigree.

Nevertheless, there are examples that highlight how the implementation of GS could benefit dairy sheep breeding programs, and in general, small populations. The Italian Sarda breed found that genomic predictions of rams were 0.13 more accurate for milk yield and 0.21 for milk fatty acid composition based on a female reference population (Usai et al., 2018; Cesarani et al., 2019a). French dairy sheep breeds used AI rams in the reference genotyped population, due to their extensive use. In the Lacaune breed, the inclusion of molecular information (compared with traditional evaluations) increased accuracies of predicted breeding values between 0.10 and 0.20 , according to the trait (Baloche et al., 2014). Similar trends were described for milk yield evaluations of Manech and Basco-Béarnaise breeds, with those being between 0.06 and 0.16 more accurate than pedigree-based evaluations (Legarra et al., 2014).

The size and characteristics of the genotyped population depends on the species and the breeding program, and its composition plays an important role in prediction accuracy. Gains in prediction accuracy and genotyped population sizes are strongly related (Daetwyler et al., 2012; Auvray et al., 2014; Schöpke and Swalve, 2016). However, breeding programs usually have limited economic resources for genotyping, making the decision to select candidates a much more cautious one. To solve this common issue, a large amount of research focuses on the design of genotyping strategies to optimize the selection of individuals to be genotyped. In this respect, there are studies based on small dairy cattle data, sheep data, simulations of the effect of selecting individuals genetically related to candidates for selection (Hayes et al., 2009; Habier et al., 2010; Clark et al., 2012), or genotyping females selected randomly, by EBV, EBV accuracy, or phenotypic value (Jiménez-Montero et al., 2012; Gao et al., 2015; Cesarani et al., 2019b). Moreover, in small dairy cattle, increasing the genotyped population size with data from other populations of the same breed, or from different but related breeds, is known to be beneficial (Lund et al., 2016; Schöpke and Swalve, 2016).

The Latxa breed is a dairy sheep breed autochthonous from the Western Pyrenees. Three strains are distinguished according to head color: Latxa Cara Rubia (LCR), Latxa Cara Negra from Euskadi (LCNEUS), and Latxa Cara Negra from Navarre (LCNNAF). Each strain has a separate breeding program. Breeding objectives are milk yield, milk composition, and udder morphology traits. The breeding program started in 1984 and is now well established, showing consolidated results with an annual genetic gain for milk yield be- tween 0.19 and 0.23 standard deviations, depending on the strain (Granado-Tajada et al., 2020).

Although GS is not implemented in Latxa breeding programs, several exploratory studies have been developed. Legarra et al. (2014) found inconsistent results when the accuracy of genomic predictions was evaluated, attributed to the distribution of genotypes across the population and the weak link between genotyped individuals with phenotypic data and candidates for selection. After some years of systematic genotyping directed toward solving these handicaps, GranadoTajada et al. (2020) looked again into the effect of including genomic information; more coherent results were found, although the inclusion of genotypes did not increase the accuracy of predictions for any of the Latxa breeds. Only AI rams were genotyped, which is a common strategy because males drive the genetic structure of the population and provide high predictive accuracy, due to the information from their daughters, as Jiménez-Montero et al. (2012) stated for dairy cattle.

Therefore, given the importance of the makeup of the genotyped population in the accuracy of genetic evaluations (Clark et al., 2012), a simulation study was designed based on the real data structure of the Latxa breeds. Thus, the aim of this work is to compare several selection strategies to form the genotyped population, in the 3 Latxa ecotypes, LCR, LCNEUS, and LCNNAF, by comparing the prediction accuracy of genomic evaluations against pedigree-based evaluations.

\section{MATERIALS AND METHODS}

\section{Genealogical, Phenotypic, and Genomic Data}

The simulation study was based on the available genealogical, phenotypic, and genomic information. Genealogical data comprised 263,308 individuals for LCNEUS, 150,185 for LCR, and 68,714 for LCNNAF. Individuals recorded into genealogy are the ones that remain in the flock ( $75 \%$ of females and $8 \%$ of males born from AI). We do not have information about the removed animals, which is especially relevant for males, due to the high selection pressure. Moreover, in the full data set, $25 \%$ of the ewes with milk records have known sire and dam, even though in data since 2000, this percentage has increased to $42 \%$. This is because the breeding program only recognizes as fathers the AI males or natural service males after paternal filiations. Details about the males and females included in pedigree and candidates to be genotyped are shown in Table 1.

Regarding phenotypic data, we used 120 d standardized milk yield information with 639,$517 ; 392,109$; and 
183,251 records of 235,360; 133,230; and 61,309 ewes for LCNEUS, LCR, and LCNNAF, respectively. The phenotypic means and standard deviations were $134 \pm$ 57 for LCNEUS, $148 \pm 68$ for LCR, and $143 \pm 57$ for LCNNAF.

The genomic data consisted of genotypes of 353 LCNEUS, 427 LCR, and 192 LCNNAF AI rams selected for milk yield, and in an effort to keep a bigger genetic diversity, animals from different families were chosen and siblings avoided. The AI rams diffuse their genetics through the population by having many daughters and being the sires of natural service rams; therefore, they are expected to be representative of each population. They were genotyped with the Illumina OvineSNP50 BeadChip (Illumina Inc.), and as quality control, markers with call rate $<0.97$ or minor allele frequency $<0.05$, and markers that were monomorphic or located in sexual chromosomes were removed, resulting in 42,547 markers. The imputation of missing genotypes was conducted with FImpute software (Sargolzaei et al., 2014).

\section{Simulation}

The simulation was carried out based on the work done by Mouresan et al. $(2017,2018)$ with a fortran 90 code, available upon request. A summary of the simulation structure is presented in Figure 1. The genomic information available was used to create the base generation. Based on these genotypes, 3 discrete generations of 1,000 individuals were generated by gene-dropping (MacCluer et al., 1986), with random mating and no sex differences, with the aim of capturing the linkage disequilibrium structure and evolve toward the overall analyzed population. Every individual had a genome of 26 chromosomes, where the 42,547 markers were distributed asymmetrically, as shown in Supplemental Table S1 (https://zenodo.org/record/ 4592995\#.YEiHibOCE2x). Out of all the markers, 5\% were randomly selected as causative mutations (QTL) from a Gaussian distribution with mean zero and variance one; a recombination rate of $1 \%$ per $\mathrm{Mb}$ was fixed. The last simulated generation was used to generate the genotypes of the founders of the real pedigree of each population. The aim of this procedure was to obtain simulated genotypes with the same genealogical structure as in the real populations, to be as close as possible to real data.

For each individual, the true breeding value (TBV) was calculated as the sum of the effects of their genotypes for each QTL. The value was rescaled by its additive standard deviation. The assumed heritability was $0.1847 \pm 0.0031$ for LCNEUS, $0.2203 \pm 0.0042$ for LCR, and $0.2225 \pm 0.0061$ for LCNNAF (GranadoTajada et al., 2020).

Phenotypic records were simulated for the individuals who already had a phenotype recorded in the real data set. Following the strategy of Mouresan et al. (2018), the simulated data reproduced the actual distribution of records across fixed and random (additive and permanent) effects. The phenotypic values of individuals were simulated by adding together the phenotypic mean, TBV, and an error term drawn from a Gaussian distribution with mean zero and variance one and rescaled by the residual standard deviation.

\section{Simulated Genotyped Population Scenarios}

To evaluate the described simulation process and to assess how close to reality the obtained results would be, a control scenario simulated the genotypes of the same individuals included in Granado-Tajada et al. (2020), keeping the same validation group. For the simulated scenarios, several variables were applied to select the individuals of the genotyped population, whereas the validation group was genotyped in all the scenarios (as described below). The selection variables considered when designing the scenarios were the sex of individu-

Table 1. Total number (and mean per year) of male and female candidates to be genotyped recorded in pedigree (1987-2017) and number with complete pedigree; males classified as AI or natural service (NSV) rams, number with offspring records, mean offspring and distribution; and mean lactation records and distribution for females

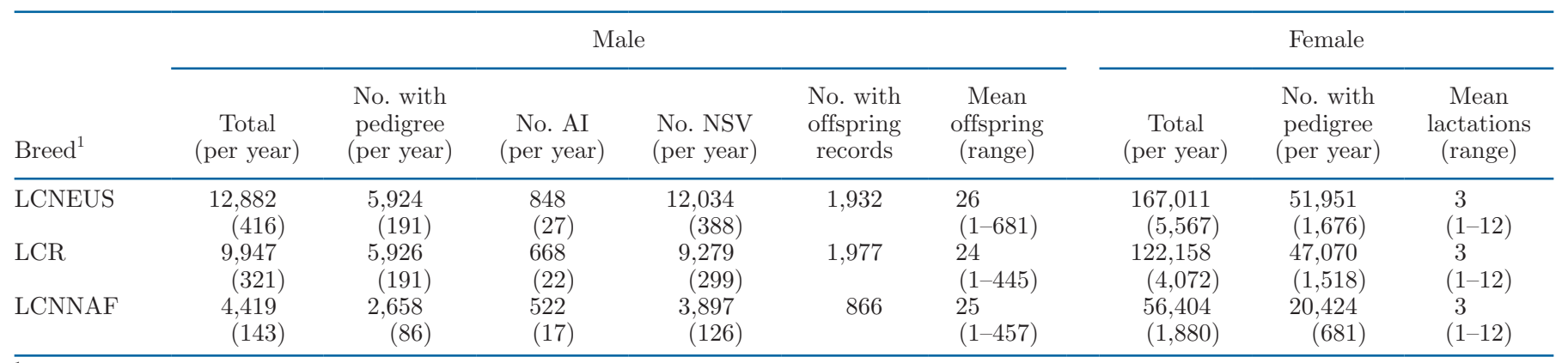

${ }^{1}$ LCNEUS = Latxa Cara Negra from Euskadi; LCR = Latxa Cara Rubia; LCNNAF = Latxa Cara Negra from Navarre. 


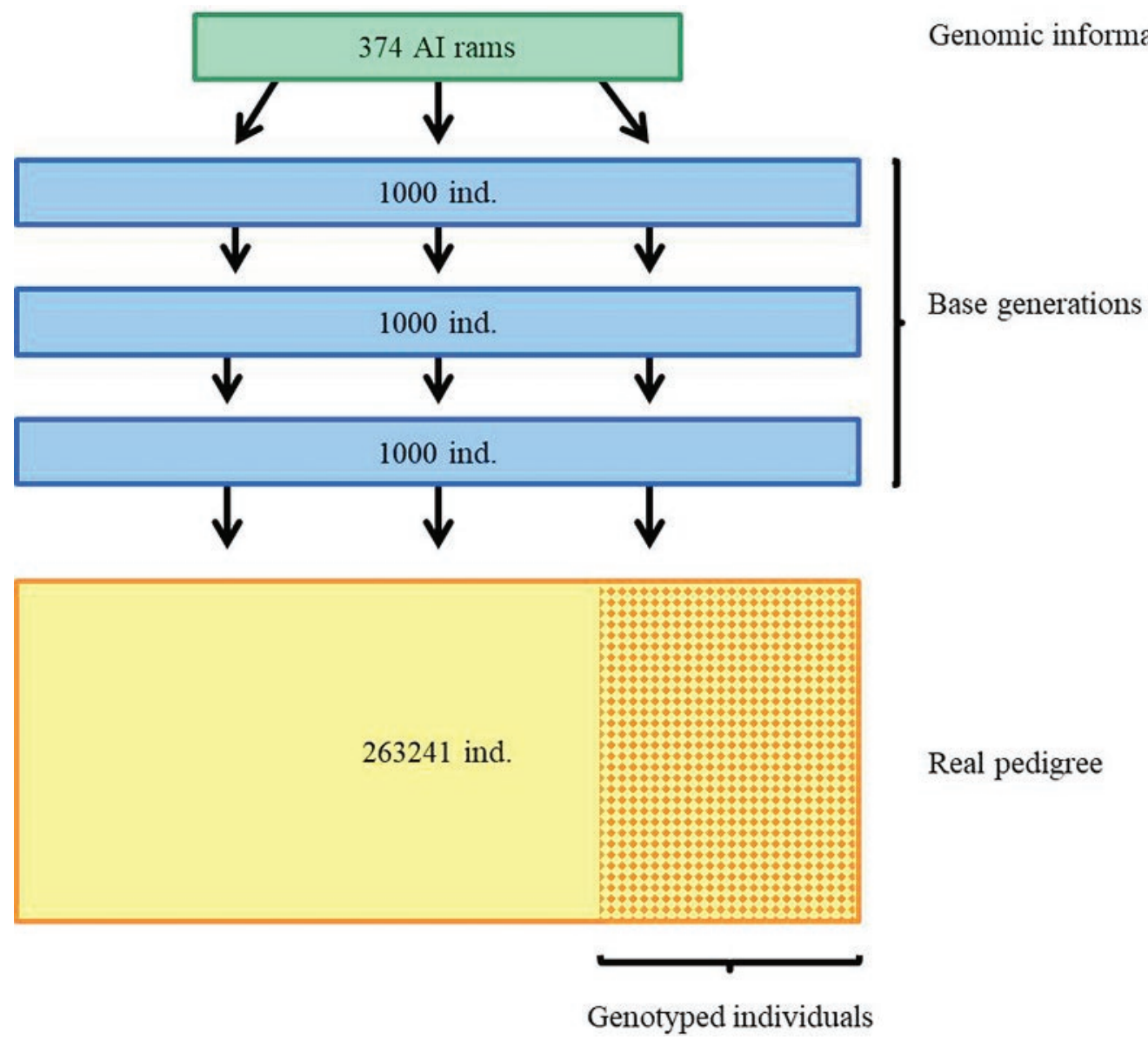

Figure 1. Structure of the simulation strategy from genomic information to the simulation of genotypes for individuals (ind.) in Latxa Cara Negra from Euskadi pedigree.

als, the EBV, pedigree knowledge, the starting year of genotyping, and the number of genotyped individuals. The selection variables used are detailed in Table 2. Each variable was defined to understand how different constitutions of the genotyped population contribute to prediction accuracy:

- Sex. Due to limited economic resources for genotyping and the higher selection pressure on males, the standard approach is to genotype males $(\mathbf{M})$. Genotyped populations comprised of females $(\mathbf{F})$ or both sexes $(\mathbf{M}+\mathbf{F})$ were considered to analyze the benefits that each sex or the combination could bring. The males selected for genotyping were not required to have daughters with phenotypic data, because in some cases, paternity is not recognized or recorded in pedigree. However, for selected females, it was compulsory to have their own phenotypic record. Selection pressure among females is low $(75 \%$ of females born from AI re- main in the flock), and almost all the ewes have recorded lactations.

- EBV. The genotyped individuals were selected based on their EBV obtained from a standard BLUP evaluation. The males were always selected by best EBV. For females, 2 variables were tested based on EBV: best ewes (B) or extreme ewes $(\mathbf{E})$. To select extreme individuals, best and worst females per year were combined in equal proportions. Because females were required to have their own records, as they are the ones selected to remain in the flock, the selected worst females are not necessarily the worst born females.

- Pedigree knowledge. Genotyping individuals with and without known pedigree (indifferent, I), and genotyping restricted to individuals with complete pedigree $(\mathbf{P})$ were tested to analyze effects of the connection with the overall population.

- Starting year. Although the breeding programs started in 1984, the start of unrestricted genotyp- 
Table 2. Selection variables to make up the genotyped population

\begin{tabular}{lllll}
\hline Selection variable & Acronym & Description & Acronym & Description \\
\hline Sex & $\mathrm{M}$ & Males & $\mathrm{F}$ & Females \\
EBV & $\mathrm{B}$ & Best EBV & $\mathrm{E}$ & Extreme EBV \\
Pedigree knowledge & $\mathrm{I}$ & Indifferent & $\mathrm{P}$ & Known \\
Starting year & - & Unrestricted & $\mathrm{R}$ & Restricted \\
Number of genotypes & $\mathrm{Max}$ & Maximum & $\%$ & Percentages \\
\hline${ }^{1}$ Both parents are known. & & & &
\end{tabular}

ing was fixed at 1987, when inseminations began. This variable allowed having a high number of genotyped animals from different generations, because the small population size could limit the potential benefits of genomic evaluations, and it meant to provide insight about future chances of the breeding program. A start restricted to 2010 (R) aimed to reflect the reality of the breeding programs (systematic genotyping started at 2010), in which the gain in prediction accuracy of genetic EBV (GEBV) could be reduced by the limited number of genotyped animals.

- Number of genotypes. To simulate scenarios close to reality, the limitations of the improvement pro- grams were considered to set a maximum number of genotyped animals per year (Max) of 300 for LCNEUS and LCR breeds and 150 for LCNNAF for each program (CONFELAC, Latxa and Carranzana Breeders' Confederation, personal communication). Moreover, the effect of genotyping fewer males per year was analyzed by testing different percentages of animals below the defined maximum (around 10, 20, 30, 50, and 70\%).

By combining these selection variables, several genotyped population scenarios were designed and are detailed in Table 3. The main strategies followed were maximum genotyping and genotyping by percentages,

Table 3. Scenarios designed by the combination of selection variables to make up the genotyped population, classified into maximum genotyping and genotyping by percentages

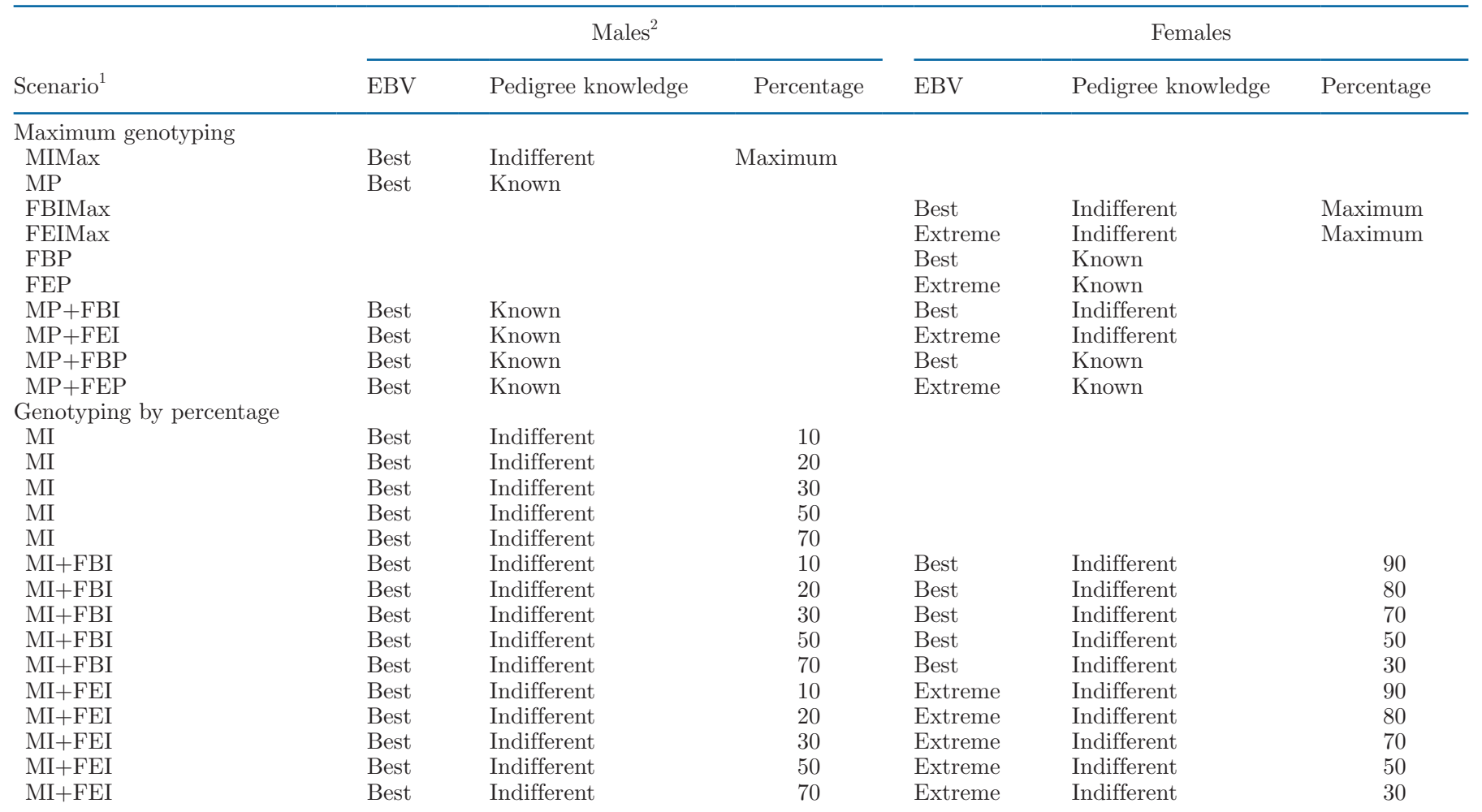

$\overline{{ }^{1} \mathrm{M}}=$ male; $\mathrm{F}$ = female; $\mathrm{I}=$ indifferent pedigree; $\mathrm{P}=$ known pedigree; $\mathrm{B}=$ genetically best individuals; $\mathrm{E}=$ combination of genetically extreme animals; Max = maximum genotyping per year; restricted scenarios follow the same strategy, but the starting year of genotyping was 2010 .

${ }^{2}$ Males born in the last year (2017) are genotyped in all scenarios. 
and all the scenarios designed by means of these strategies were also simulated with restricted start of genotyping to 2010 (scenario acronym $+\mathbf{R}$ ).

\section{Estimated Breeding Values and Validation}

The phenotypic and genotypic data obtained were used to calculate EBV, by pedigree-based BLUP; and GEBV, using single-step genomic BLUP (ssGBLUP; Aguilar et al., 2010; Christensen and Lund, 2010). In both cases the model for milk yield was as follows:

$$
Y_{i j k l m}=(F Y S)_{i}+A_{j}+I_{k}+L_{l}+u_{m}+p_{m}+e_{i j k l m},
$$

where flock-year-season $(F Y S)$, age-parity number $(A)$, interval lambing-first milk recording $(I)$, and number of alive lambs born $(L)$ were included as fixed effects (Legarra et al., 2005), with 19,062, 11, 8, and 3 levels for LCNEUS; 10,908, 10, 8, and 3 levels for LCR; and $4,800,9,8$, and 3 levels for LCNNAF, respectively. In addition, additive genetic $(u)$ and individual random environmental or permanent $(p)$ were considered as random effects. Both analyses were performed using the BLUPf90 software suite (Misztal et al., 2002) with the default quality control values.

The different genotyping strategies were compared in terms of relative difference in accuracy over pedigree evaluations, estimated as the Pearson correlation between TBV and estimated breeding values (EBV and GEBV) of the validation group, made up of the males born in the previous year (2017). These validation individuals were considered the actual candidates to selection; they do not have daughters with records and were genotyped in all the scenarios (298 for LCNEUS; 287 for LCR; 126 for LCNNAF). Each one of the genotyping scenarios described was replicated 20 times, and we present the mean and standard error of the relative accuracy difference.

\section{RESULTS AND DISCUSSION}

In a preliminary approach, a control scenario was carried out to mimic the study done with real data (Granado-Tajada et al., 2020). In the control scenario, the accuracy difference between genomic and pedigreebased evaluations was $0.0113 \pm 0.0030$ for LCNEUS and $0.0017 \pm 0.0015$ for LCR. The real data study described accuracy differences of $0.0097 \pm 0.0423$ for LCNEUS and $0.0064 \pm 0.0387$ for LCR. The LCNNAF was not included in the study mentioned, due to the limited number of genotyped individuals. The results are not directly comparable because the current work compared predicted breeding values with TBV, and the previous study was a cross validation in which the metafounder theory (Legarra et al., 2015) was used; however, similar results were obtained, reinforcing the validity of the simulation approach.

The definition of the genotyped population is a determining factor for prediction accuracy under genomic evaluation. To analyze different ways to select animals for the genotyped population, various combinations of sex of individuals, EBV, pedigree knowledge, starting year of genotyping, and number of genotyped individuals were tested. In all cases, breeding values estimated by ssGBLUP showed higher accuracies (mean 0.5841 for LCNEUS, 0.5981 for LCR, 0.5507 for LCNNAF) than pedigree-based BLUP evaluations (mean 0.4570 for LCNEUS, 0.5190 for LCR, 0.5111 for LCNNAF), so the mean relative accuracy differences were always positive.

\section{Maximum Genotyping}

Regarding the scenarios designed by genotyping the maximum number of individuals, results of the relative accuracy difference of genomic evaluations in contrast to pedigree-based evaluations are summarized in Figure 2 and Supplemental Tables S2-S7 (https://zenodo.org/ record/4592995\#.YEiHibOCE2x). Broadly speaking, the genotyped population comprised of only females provided the highest relative accuracies. These higher accuracies of genomic predictions were more relevant in unrestricted data sets (Figure 2, left). In restricted data sets (Figure 2, right), genotyped population of only females brought similar accuracies to genotyped populations with both sexes. When only males made up the genotyped population, lower relative accuracies were shown in both unrestricted and restricted data sets. Genotyping exclusively individuals with known pedigree, with the consequent reduction in number, showed a noticeable reduction in accuracy in female-only unrestricted scenarios. These results highlighted that when there is a considerable proportion of unknown pedigree, as in our case (around $45 \%$ for males and $65 \%$ for females), the increase in accuracy of ssGBLUP is strengthened by the incorporation of genotyped females with their own records, rather than genotyping unconnected males.

Regarding unrestricted scenarios (Figure 2, left), when only females were genotyped, the effect of selecting the best ewes or extreme ewes by EBV with indifferent pedigree (FBIMax and FEIMax) was tested. Both alternatives showed quite similar relative accuracy differences (e.g., for LCNEUS 0.5369 and 0.5492, respectively). When only females with complete pedigree were genotyped (FBP and FEP), the relative accuracy difference diminished $(0.3657$ and 0.3789 , respectively, for LCNEUS), even though the number of genotyped 
females per year was much lower (around $50 \%$ less), and the mean number of lactations per genotyped ewe kept constant around 3. Similar trends were shown for LCR and LCNNAF breeds, with smaller relative accuracy differences (e.g., FBIMax and FBP were 0.3322 and 0.2375 for LCR, and 0.1252 and 0.1447 for LCNNAF, respectively). There is a loss in accuracy of GEBV when the number of genotyped females decreases, which is less noticeable according to genotyped population size. This is probably due to the fact that although these individuals do not have complete pedigree, their own records, and possibly the data and connections of their offspring, provide useful information for more accurate evaluations.

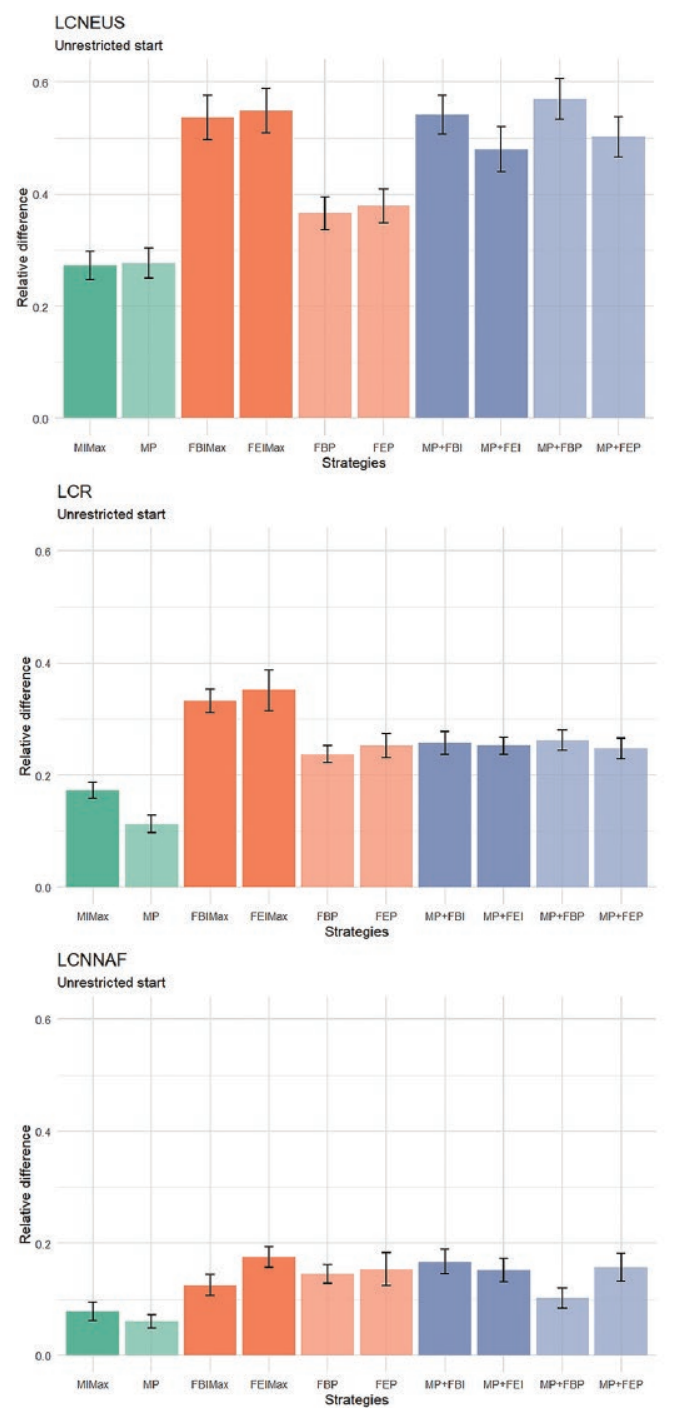

Similar results found between scenarios genotyping best or extreme females could be due to some degree of selection, because genotyped females were required to have their own records. Therefore, these individuals have already been selected to remain at the flock. The applicability of genotyped populations formed exclusively by females has been investigated in the literature, and some studies describe better accuracies than pedigree index (Ding et al., 2013) or genotyped population of males (Jiménez-Montero et al., 2012).

Genotyped populations of only males have been the standard approach since the beginning of GS (VanRaden, 2020) due to limited economic resources, because the selection pressure exerted on this sex is higher, and

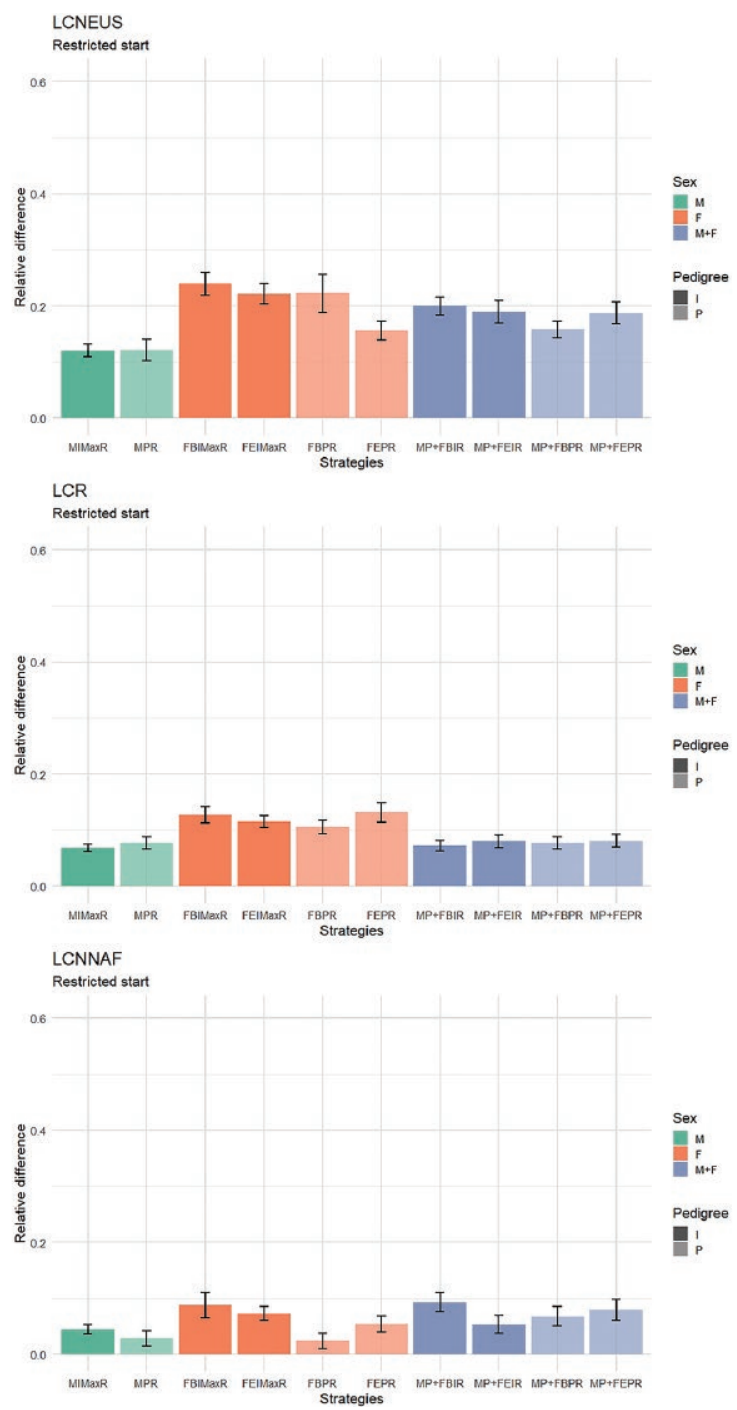

Figure 2. Relative accuracy difference of genomic evaluations in contrast to pedigree-based evaluations, comparing unrestricted (left) and restricted (right) start of genotyping into different genotyping strategies for 3 breeds. $\mathrm{M}=$ males; $\mathrm{F}=$ females; $\mathrm{M}+\mathrm{F}=$ complementation of males with complete pedigree with females; $\mathrm{B}=$ genetically best individuals; $\mathrm{E}=$ combination of genetically extreme animals; $\mathrm{I}=$ indifferent pedigree knowledge; $\mathrm{P}$ = only individuals with complete pedigree; Max = maximum genotyping per year; $\mathrm{R}=$ restricted start of genotyping; LCNEUS = Latxa Cara Negra from Euskadi; LCR = Latxa Cara Rubia; and LCNNAF = Latxa Cara Negra from Navarre. Bars indicate standard error. 
the diffusion of the genetics of rams achieved by AI is larger. When all the efforts focused on genotyping the best males (indifferent pedigree) per year up to the stabilized maximum number of individuals (MIMax) in the unrestricted scenarios (Figure 2, left), a relative accuracy difference of 0.2729 was achieved for LCNEUS. The genotyped males in this breed had a mean number of daughters in data of 3.3. Selecting among those best males the ones with complete pedigree (MP) reduced the number of genotyped males to around 130 animals per year in LCNEUS, but the gain in accuracy was kept at 0.2769 , and the mean number of daughters in data increased to 6.7. Similar trends were shown for LCR and LCNNAF breeds, with smaller relative accuracy differences (MIMax and MP were 0.1729 and 0.1131, respectively, for LCR; and 0.0777 and 0.0607 for LCNNAF). By contrast to female genotyping, the relative accuracy difference decrease was slight, whereas the number of genotyped rams was notably reduced (even $50 \%$ ), and the average number of daughters in data was increased (around 150\%), which reflects the importance of genotyping animals well connected in the population. These results confirmed that the genotyping of rams in flocks have a limited effect on prediction accuracy if they are isolated in the pedigree and without offspring records (Pszczola et al., 2012; Shabalina et al., 2017; de Oliveira et al., 2019).

Regarding the scenarios composed of both sexes (males with complete pedigree combined with females) in unrestricted data set (Figure 2, left), intermediate results between only female and only male genotyping were found. As in the case of only female genotyped scenarios, a similar relative accuracy difference was found regardless of the strategy used to select the genotyped females. Moreover, females with best EBV $(\mathrm{MP}+\mathrm{FBI})$ or with extreme EBV $(\mathrm{MP}+\mathrm{FEI})$ showed almost the same relative accuracy difference as females with complete pedigree $(\mathrm{MP}+\mathrm{FBP}$ and $\mathrm{MP}+\mathrm{FEP})$. Mean relative accuracy difference of these scenarios was 0.5234 for LCNEUS, 0.2549 for LCR, and 0.1445 for LCNNAF.

The inclusion of females in the genotyped population was also found to be beneficial in species where the effect of female paths on genetic progress is strong, and the effect of genomic data on accuracy depends on the existing population structure (Lourenco et al., 2015). The effect of genotyped populations including female genomic information on genomic prediction accuracy has been thoroughly studied (Koivula et al., 2016; Uemoto et al., 2017; Perez et al., 2019), and also as a strategy to increase the genotyped population size into small populations (Jiménez-Montero et al., 2012; Lund et al., 2016; Jenko et al., 2017). Different strategies have been proposed to assess an optimal selection of individ- uals to be genotyped. Including genomic information of females selected based on a divergent strategy seems to be better than a random or directional approach (Jenko et al., 2017; Perez et al., 2019), and avoiding the inclusion of only selected individuals would reduce prediction bias (Vitezica et al., 2011; Koivula et al., 2016). In practical terms not all the strategies are feasible for animal breeding programs and even less for small populations. Including the genotypes of high yield females by proportional sampling within detected communities could help to obtain more value from genotypes and phenotypes (Perez et al., 2019).

As the simulation was based on real data, it should be considered that the results obtained are likely to be conditioned by the individuals recorded into pedigree. Only animals that have been selected to remain in the flock are recorded, and there is no information about the ones removed. This is especially relevant for males, because the high selection pressure results in only a few selected individuals recorded in the pedigree. Genotyping males before selection could bring different results, as other studies have shown (Lourenco et al., 2015).

Finally, maximum genotyping scenarios were also simulated under restricted genotyping starting year $($ scenario $+\mathrm{R})$ and relative accuracy difference results are also summarized in Figure 2 (right). Starting the genotyping in 2010 caused a drop in relative accuracy difference in all the scenarios, and trends were not as clear as in unrestricted scenarios. When the maximum number of males per year was genotyped (MIMaxR), the relative accuracy difference for LCNEUS was 0.1206 , genotyping only best females (FBIMaxR) was 0.2393 , and genotyping extreme females was 0.2217 . Compared with unrestricted scenarios, this reduction in relative accuracy difference was probably due to the reduction of the genotyped population size by almost $60 \%$, removing relatives of past generations. When only the animals with complete pedigrees were considered, the relative accuracy difference was also reduced, although in a smaller proportion than in unrestricted scenarios. When males with complete pedigree were combined with females, the relative accuracy difference dropped by $60 \%$ compared with unrestricted scenarios regardless of the applied strategy to select females.

At this moment, the restricted scenarios are closer to the reality of Latxa breeding programs than unrestricted ones. So, the expected gain in accuracy in the short term for Latxa populations would be around $11 \%$. The lower results showed by restricted scenarios $(60 \%$ less accuracy difference than unrestricted scenarios), highlighted the relevance of having genomic data from various previous generations.

Clear examples of recent implementation of GS with gains in accuracy over pedigree-based estimates are 
French dairy sheep breeds. Lacaune and Manech breeds have genotyped rams since the 1990s, and these breeding programs implemented a genomic scheme in 2015 and 2017, respectively (Baloche et al., 2014; Legarra et al., 2014). By that date, each program had genotyped more than 1,900 and 1,000 individuals, respectively; a genotyped population size over the minimum of 1,000 individuals set by Shumbusho et al. (2013) to achieve benefits from GS over classical selection in dairy sheep. The inclusion of molecular information was described to increase prediction accuracies of milk yield evaluations 0.15 for Lacaune, and 0.16 for Manech Tête Rousse (Baloche et al., 2014; Legarra et al., 2014). These gains in accuracy were similar to the found in Latxa populations when the genotyped of between 1,000 and 2,000 individuals were simulated.

Before implementing GS, the balance between economic cost and benefit should be considered, more complex to reach equilibrium for small ruminant breeding programs. However, simulation studies done for dairy and meat sheep concluded that an optimum balance exists by implementing some strategies to take advantage from a GS scheme (Buisson et al., 2014; Shumbusho et al., 2016). Regarding the Latxa breeding program, it would be interesting to analyze in detail specific costs and benefits of the genotyping strategies.

\section{Genotyping by Percentages}

Seeking a balance between prediction accuracy and economic costs, the effect of genotyping a lower number of individuals per year was analyzed by testing different percentages of animals below the maximum number of each breeding program (300 individuals for LCNEUS and LCR or 150 individuals for LCNNAF). Relative accuracy difference results of genomic evaluations in contrast to pedigree-based evaluations are summarized in Figure 3 and Supplemental Tables S8-S13 (https: //zenodo.org/record/4592995\#.YEiHibOCE2x). In unrestricted scenarios genotyping only males with indifferent pedigree (MI; Figure 3, left), as might be expected (Meuwissen et al., 2001), the relative accuracy difference rose according to the number of genotyped individuals in the 3 breeds. An increment of $60 \%$ was achieved from 10 to $70 \%$ male genotyping scenarios. By contrast, by genotyping $70 \%$ of males per year, similar relative accuracy difference to that achieved with $100 \%$ of the maximum number of genotyped males (MIMax) was found. When genotyping the maximum number of males, the relative accuracy difference achievable was around 0.3049 for LCNEUS, 0.1157 for LCR, and 0.0834 for LCNNAF. From $30 \%$ of genotyped males, the relative accuracy difference increase was less notice- able (except for LCNEUS), even though the size of the genotyped population kept increasing.

When those percentages of genotyped males (MI from 10-70\%) were combined with females (FBI or FEI) up to the maximum number of genotyped animals per year to make up different proportions of sexes (Figure 3, left), similar relative accuracy differences were found in all the scenarios (best or extreme EBV females). This genotyping scenario showed the highest mean relative accuracy differences, around 0.4999 for LCNEUS, 0.2848 for LCR, and 0.1259 for LCNNAF, which were similar to the results shown on Figure 2 for scenarios with both sexes into the genotyped population. Once again, the benefit of combining males and females into genotyped populations is reflected in prediction accuracy.

The genotyping of different percentages of animals and varying proportions of sexes were also simulated with a restricted start of genotyping, and relative accuracy difference results of genomic predictions comparing with pedigree-based ones are shown in Figure 3 (right). As in previous results, the relative accuracy difference was lower than for unrestricted scenarios, but always keeping the same trends and the combination of both sexes in the genotyped population showed the higher relative accuracy differences.

From an overall perspective of the simulated scenarios and given the fixed genotyping maximum, there appeared to be a limit to the relative accuracy difference achievable, regardless of the genotyping strategy applied, which for LCNEUS was at 0.56, for LCR at 0.33 , and for LCNNAF at 0.18. The relative accuracy difference was different depending on the breed. The LCNEUS had the possibility to achieve the biggest benefits from the genomic information, with a mean relative accuracy difference for unrestricted scenarios of 0.4239 , whereas LCR had a mean relative accuracy difference of 0.2324 and LCNNAF of 0.1153 . These differences could be due to the population size and the amount and characteristics of the recorded information. LCNEUS breed had the biggest amount of data, but compared with LCR, there was no structural differences because the use of AI, knowledge of genealogy and the distribution of offspring and lactations were similar in both programs. Meanwhile LCNNAF was the smaller population and the breeding program started later, so the gain in accuracy was limited. Despite the different mean relative accuracy differences, the 3 breeds behave similarly.

Restricted scenarios are closer to the current reality of the breeding program and, in spite of lower mean relative accuracy differences (LCNEUS 0.1684; LCR 0.0883; and LCNNAF 0.0560), genomic predictions 
were shown to be more accurate than pedigree-based predictions. These gains in prediction accuracy would be very interesting, given that the average accuracy is 0.55 for LCNEUS, 0.50 for LCR, and 0.60 for LCNNAF (Granado-Tajada et al., 2020). Moreover, previous studies did not find increases in prediction accuracy by the genotyping of AI rams (Legarra et al., 2014; Granado-Tajada et al., 2020). So, although accuracy gains yield by simulation is known to be bigger than that reported in real data studies (Legarra et al., 2008; de Roos et al., 2009), the results showed a potential benefit of implementing a GS scheme in Latxa breeds,
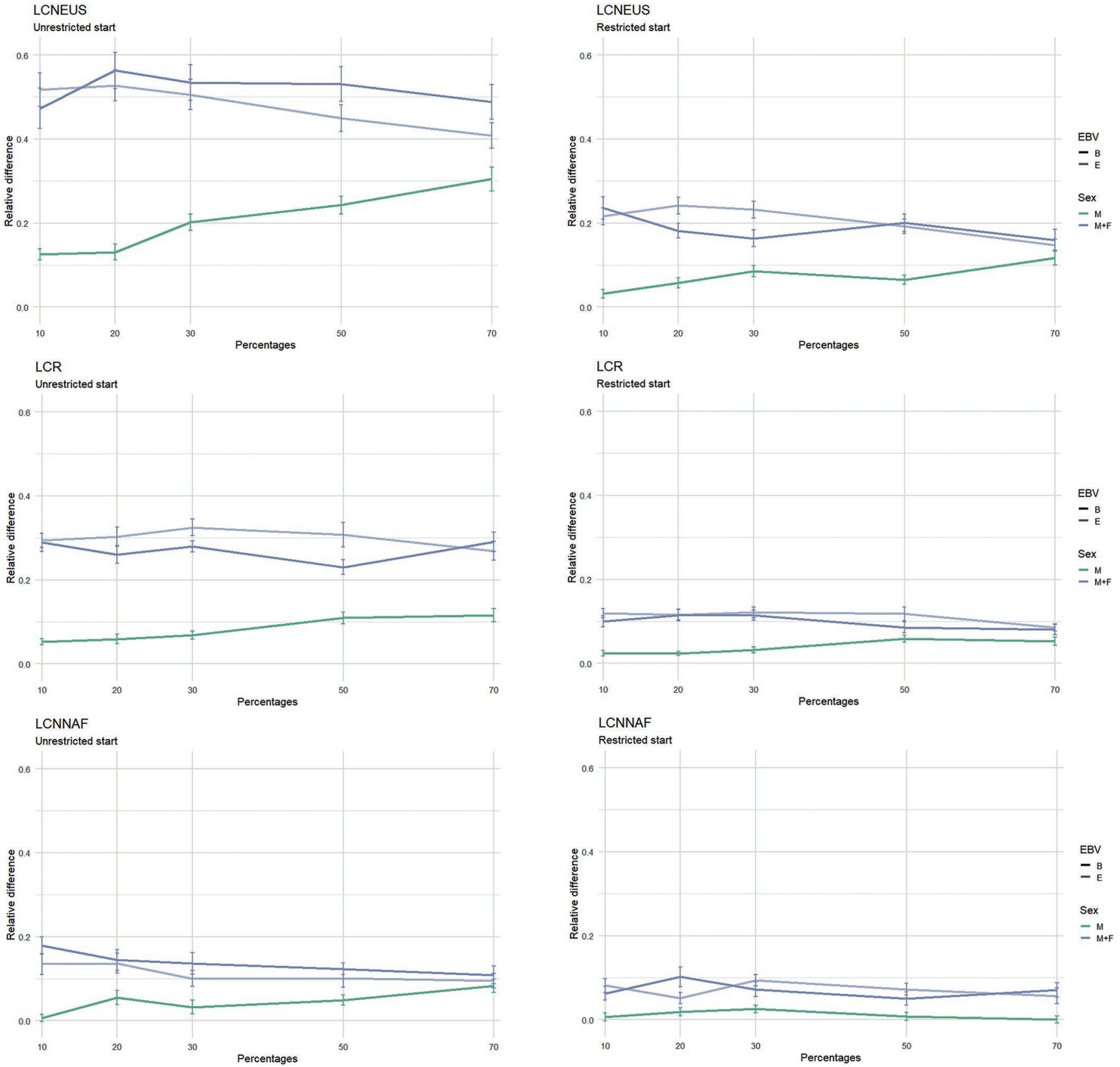

Figure 3. Effect of various combinations of genotyped individual percentages on the relative accuracy difference of genomic evaluations in contrast to pedigree-based evaluations, for unrestricted (left) and restricted (right) start of genotyping of different genotyping strategies. $\mathrm{M}$ $=$ males; $\mathrm{M}+\mathrm{F}=$ complementation of males with females; $\mathrm{B}=$ genetically best individuals; $\mathrm{E}=$ combination of genetically extreme animals; LCNEUS = Latxa Cara Negra from Euskadi; LCR = Latxa Cara Rubia; and LCNNAF = Latxa Cara Negra from Navarre. Bars indicate standard error. 
and go in depth into the knowledge of GS applied to small populations.

\section{CONCLUSIONS}

The studied genotyped population scenarios showed that breeding values estimated by ssGBLUP were more accurate than pedigree-based BLUP evaluations, reflecting a potential benefit in terms of prediction accuracy of implementing a GS scheme in Latxa breeding programs. Genotyping males was found to be beneficial, when they are not isolated individuals and they have daughters with phenotypic records. Genotyping females with their own lactation records was shown to increase prediction accuracy, regardless of their connection level. No differences were found between the genotyping of genetically best or extreme individuals, probably due to the low selection pressure on females. The combined genotyping of males and females provided intermediate accuracies regardless of the female selection strategy. The importance of genotyping individuals from various generations has been highlighted, although without historical individuals it is also possible to take advantage of accurate genomic predictions. In the same way, larger genotyped population sizes led to greater relative accuracy differences, but by only genotyping males, a maximum relative difference was achievable. Therefore, genotyping rams is of interest, but at some point, it would be more beneficial to focus the efforts of the breeding program on combining males with females. Regarding Latxa breeding programs, the implementation of GS seems to be beneficial and the implementation of a genotyped population of both sexes would be worthwhile.

\section{ACKNOWLEDGMENTS}

The authors thank the Confederación de Asociaciones de Criadores de Ovino de Razas Latxa y Carranzana (CONFELAC, Vitoria-Gasteiz, Spain) for providing the milk recording and pedigree data. This work would not have been possible without the funding of the Department of Economic Development and Infrastructure of the Basque Government, with the grant for young researchers and technologists; and the support of the project ARDI (EFA208/16), funded by INTERREG POCTEFA with the European Fund for Regional Development (FEDER). The authors have no conflicts of interest.

\section{REFERENCES}

Aguilar, I., I. Misztal, D. L. Johnson, A. Legarra, S. Tsuruta, and T. J. Lawlor. 2010. Hot topic: A unified approach to utilize phenotypic, full pedigree, and genomic information for genetic evaluation of Holstein final score. J. Dairy Sci. 93:743-752. https://doi.org/10 $.3168 /$ jds.2009-2730.

Auvray, B., J. C. McEwan, S.-A. N. Newman, M. Lee, and K. G. Dodds. 2014. Genomic prediction of breeding values in the New Zealand sheep industry using a 50K SNP chip. J. Anim. Sci. 92:4375-4389. https://doi.org/10.2527/jas.2014-7801.

Baloche, G., A. Legarra, G. Sallé, H. Larroque, J. M. Astruc, C. Robert-Granié, and F. Barillet. 2014. Assessment of accuracy of genomic prediction for French Lacaune dairy sheep. J. Dairy Sci. 97:1107-1116. https://doi.org/10.3168/jds.2013-7135.

Boichard, D., V. Ducrocq, P. Croiseau, and S. Fritz. 2016. Genomic selection in domestic animals: Principles, applications and perspectives. C. R. Biol. 339:274-277. https://doi.org/10.1016/j.crvi 2016.04.007.

Buisson, D., G. Lagriffoul, G. Baloche, X. Aguerre, P. Boulenc, F. Fidelle, G. Frégeat, B. Giral-Viala, P. Guibert, P. Panis, C. Soulas, J. M. Astruc, and F. Barillet. 2014. Genomic breeding schemes in French Lacaune and Manech dairy sheep: Design and expected genetic gain. In Proceedings of the 10th World Congress of Genetics Applied to Livestock Production, Vancouver, Canada.

Cesarani, A., G. Gaspa, F. Correddu, M. Cellesi, C. Dimauro, and N. P. P. Macciotta. 2019a. Genomic selection of milk fatty acid composition in Sarda dairy sheep: Effect of different phenotypes and relationship matrices on heritability and breeding value accuracy. J. Dairy Sci. 102:3189-3203. https://doi.org/10.3168/jds .2018-15333.

Cesarani, A., I. Pocrnic, N. P. P. Macciotta, B. O. Fragomeni, I. Misztal, and D. A. L. Lourenco. 2019b. Bias in heritability estimates from genomic restricted maximum likelihood methods under different genotyping strategies. J. Anim. Breed. Genet. 136:40-50. https://doi.org/10.1111/jbg.12367.

Christensen, O. F., and M. S. Lund. 2010. Genomic prediction when some animals are not genotyped. Genet. Sel. Evol. 42:2. https:// doi.org/10.1186/1297-9686-42-2.

Clark, S. A., J. M. Hickey, H. D. Daetwyler, and J. H. van der Werf. 2012. The importance of information on relatives for the prediction of genomic breeding values and the implications for the makeup of reference data sets in livestock breeding schemes. Genet. Sel. Evol. 44:4. https://doi.org/10.1186/1297-9686-44-4.

Daetwyler, H. D., A. A. Swan, J. H. J. van der Werf, and B. J. Hayes. 2012. Accuracy of pedigree and genomic predictions of carcass and novel meat quality traits in multi-breed sheep data assessed by cross-validation. Genet. Sel. Evol. 44:33. https://doi.org/10.1186/ 1297-9686-44-33.

de Oliveira, H. R., L. F. Brito, M. Sargolzaei, F. F. E. Silva, J. Jamrozik, D. A. L. Lourenco, and F. S. Schenkel. 2019. Impact of including information from bulls and their daughters in the training population of multiple-step genomic evaluations in dairy cattle: A simulation study. J. Anim. Breed. Genet. 136:441-452. https://doi .org/10.1111/jbg.12407.

de Roos, A. P. W., B. J. Hayes, and M. E. Goddard. 2009. Reliability of genomic predictions across multiple populations. Genetics 183:1545-1553. https://doi.org/10.1534/genetics.109.104935.

Ding, X., Z. Zhang, X. Li, S. Wang, X. Wu, D. Sun, Y. Yu, J. Liu, Y. Wang, Y. Zhang, S. Zhang, Y. Zhang, and Q. Zhang. 2013. Accuracy of genomic prediction for milk production traits in the Chinese Holstein population using a reference population consisting of cows. J. Dairy Sci. 96:5315-5323. https://doi.org/10.3168/ jds.2012-6194.

Gao, H., P. Madsen, U. S. Nielsen, G. P. Aamand, G. Su, K. Byskov, and J. Jensen. 2015. Including different groups of genotyped females for genomic prediction in a Nordic Jersey population. J. Dairy Sci. 98:9051-9059. https://doi.org/10.3168/jds.2015-9947.

Granado-Tajada, I., A. Legarra, and E. Ugarte. 2020. Exploring the inclusion of genomic information and metafounders in Latxa dairy sheep genetic evaluations. J. Dairy Sci. 103:6346-6353. https://doi .org/10.3168/jds.2019-18033.

Habier, D., J. Tetens, F. R. Seefried, P. Lichtner, and G. Thaller. 2010. The impact of genetic relationship information on genomic breeding values in German Holstein cattle. Genet. Sel. Evol. 42:5. https: //doi.org/10.1186/1297-9686-42-5. 
Hayes, B. J., P. M. Visscher, and M. E. Goddard. 2009. Increased accuracy of artificial selection by using the realized relationship matrix. Genet. Res. (Camb) 91:47-60. https://doi.org/10.1017/ S0016672308009981.

Ibañez-Escriche, N., and O. Gonzalez-Recio. 2011. Review. Promises, pitfalls and challenges of genomic selection in breeding programs. Span. J. Agric. Res. 9:404-413. https://doi.org/10.5424/sjar/ 20110902-447-10.

Ibañez-Escriche, N., and H. Simianer. 2016. From the editors: Animal breeding in the genomics era. Anim. Front. 6:4-5. https://doi.org/ 10.2527/af.2016-0001.

Jenko, J., G. R. Wiggans, T. A. Cooper, S. A. E. Eaglen, W. G. L. Luff, M. Bichard, R. Pong-Wong, and J. A. Woolliams. 2017. Cow genotyping strategies for genomic selection in a small dairy cattle population. J. Dairy Sci. 100:439-452. https://doi.org/10.3168/jds 2016-11479.

Jiménez-Montero, J. A., O. González-Recio, and R. Alenda. 2012. Genotyping strategies for genomic selection in small dairy cattle populations. Animal 6:1216-1224. https://doi.org/10.1017/ S1751731112000341.

Kijas, J. W., L. Porto-Neto, S. Dominik, A. Reverter, R. Bunch, R. McCulloch, B. J. Hayes, R. Brauning, and J. McEwan. 2014. Linkage disequilibrium over short physical distances measured in sheep using a high-density SNP chip. Anim. Genet. 45:754-757. https:// doi.org/10.1111/age.12197.

Koivula, M., I. Strandén, G. P. Aamand, and E. A. Mäntysaari. 2016. Effect of cow reference group on validation reliability of genomic evaluation. Animal 10:1061-1066. https://doi.org/10.1017/ S1751731115002864.

Legarra, A., G. Baloche, F. Barillet, J. M. Astruc, C. Soulas, X. Aguerre, F. Arrese, L. Mintegi, M. Lasarte, F. Maeztu, I. Beltrán de Heredia, and E. Ugarte. 2014. Within- and across-breed genomic predictions and genomic relationships for Western Pyrenees dairy sheep breeds Latxa, Manech, and Basco-Béarnaise. J. Dairy Sci. 97:3200-3212. https://doi.org/10.3168/jds.2013-7745.

Legarra, A., O. F. Christensen, Z. G. Vitezica, I. Aguilar, and I. Misztal. 2015. Ancestral relationships using metafounders: Finite ancestral populations and across population relationships. Genetics 200:455-468. https://doi.org/10.1534/genetics.115.177014.

Legarra, A., P. López-Romero, and E. Ugarte. 2005. Bayesian model selection of contemporary groups for BLUP genetic evaluation in Latxa dairy sheep. Livest. Prod. Sci. 93:205-212. https://doi.org/ 10.1016/j.livprodsci.2004.10.008.

Legarra, A., C. Robert-Granié, E. Manfredi, and J. M. Elsen. 2008. Performance of genomic selection in mice. Genetics 180:611-618. https://doi.org/10.1534/genetics.108.088575.

Lourenco, D. A. L., B. O. Fragomeni, S. Tsuruta, I. Aguilar, B. Zumbach, R. J. Hawken, A. Legarra, and I. Misztal. 2015. Accuracy of estimated breeding values with genomic information on males, females, or both: An example in broiler chicken. Genet. Sel. Evol. 47:56. https://doi.org/10.1186/s12711-015-0137-1.

Lund, M. S., I. van den Berg, P. Ma, R. F. Brøndum, and G. Su. 2016. How to improve genomic predictions in small dairy cattle populations. Animal 10:1042-1049. https://doi.org/10.1017/ S1751731115003031.

MacCluer, J. W., J. L. VandeBurg, B. Read, and O. A. Ryder. 1986. Pedigree analysis by computer simulation. Zoo Biol. 5:147-160. https://doi.org/10.1002/zoo.1430050209.

Meuwissen, T. H. E., B. J. Hayes, and M. E. Goddard. 2001. Prediction of total genetic value using genome-wide dense marker maps. Genetics 157:1819-1829.

Misztal, I., S. Tsuruta, T. Strabel, B. Auvray, T. Druet, and D. H. Lee. 2002. BLUPF90 and related programs (BGF90). In Proceedings of the 7th World Congress on Genetics Applied to Livestock Production, Aug. 19-23, Montpellier, France.

Mouresan, E. F., J. Altarriba, C. Moreno, S. Munilla, A. GonzálezRodríguez, and L. Varona. 2017. Performance of genomic selection under a single-step approach in autochthonous Spanish beef cattle populations. J. Anim. Breed. Genet. 134:289-299. https://doi.org/ 10.1111/jbg.12253.
Mouresan, E. F., J. J. Cañas-Álvarez, A. González-Rodríguez, S. Munilla, J. Altarriba, C. Díaz, J. A. Baró, A. Molina, J. Piedrafita, and L. Varona. 2018. Evaluation of the potential use of a meta-population for genomic selection in autochthonous beef cattle populations. Animal 12:1350-1357. https://doi.org/10.1017/ S175173111700283X.

Perez, B. C., J. C. C. Balieiro, R. Carvalheiro, F. Tirelo, G. A. Oliveira Junior, J. M. Dementshuk, J. P. Eler, J. B. S. Ferraz, and R. V. Ventura. 2019. Accounting for population structure in selective cow genotyping strategies. J. Anim. Breed. Genet. 136:23-39. https://doi.org/10.1111/jbg.12369.

Pszczola, M., T. Strabel, J. A. M. van Arendonk, and M. P. L. Calus. 2012. The impact of genotyping different groups of animals on accuracy when moving from traditional to genomic selection. J. Dairy Sci. 95:5412-5421. https://doi.org/10.3168/jds.2012-5550.

Rupp, R., S. Mucha, H. Larroque, J. McEwan, and J. Conington. 2016. Genomic application in sheep and goat breeding. Anim. Front. 6:39-44. https://doi.org/10.2527/af.2016-0006.

Sargolzaei, M., P. Chesnais, and F. S. Schenkel. 2014. A new approach for efficient genotype imputation using information from relatives. BMC Genomics 15:478. https://doi.org/10.1186/1471-2164-15 -478 .

Schöpke, K., and H. H. Swalve. 2016. Review: Opportunities and challenges for small populations of dairy cattle in the era of genomics. Animal 10:1050-1060. https://doi.org/10.1017/ S1751731116000410.

Shabalina, T., E. C. G. Pimentel, C. Edel, L. Plieschke, R. Emmerling, and K.-U. Götz. 2017. Short communication: The role of genotypes from animals without phenotypes in single-step genomic evaluations. J. Dairy Sci. 100:8277-8281. https://doi.org/10.3168/jds 2017-12734.

Shumbusho, F., J. Raoul, J. M. Astruc, I. Palhiere, and J. M. Elsen. 2013. Potential benefits of genomic selection on genetic gain of small ruminant breeding programs. J. Anim. Sci. 91:3644-3657. https://doi.org/10.2527/jas.2012-6205.

Shumbusho, F., J. Raoul, J. M. Astruc, I. Palhiere, S. Lemarié, A. Fugeray-Scarbel, and J. M. Elsen. 2016. Economic evaluation of genomic selection in small ruminants: A sheep meat breeding program. Animal 10:1033-1041. https://doi.org/10.1017/ S1751731115002049.

Uemoto, Y., T. Osawa, and J. Saburi. 2017. Effect of genotyped cows in the reference population on the genomic evaluation of Holstein cattle. Animal 11:382-393. https://doi.org/10.1017/ S1751731116001762.

Usai, M. G., S. Salaris, S. Casu, T. Sechi, S. Miari, P. Carta, and A. Carta. 2018. Feasibility of genomic predictions of Sarda breed rams using a female reference population. In Proceedings of the 11th World Congress on Genetics Applied to Livestock Production, Auckland, New Zealand.

van den Berg, I., T. H. E. Meuwissen, I. M. MacLeod, and M. E. Goddard. 2019. Predicting the effect of reference population on the accuracy of within, across, and multibreed genomic prediction. J. Dairy Sci. 102:3155-3174. https://doi.org/10.3168/jds.2018-15231.

VanRaden. P. M. 2020. Symposium review: How to implement genomic selection. J. Dairy Sci. 103:5291-5301. https://doi.org/10 $.3168 /$ jds.2019-17684.

VanRaden, P. M., C. P. Van Tassell, G. R. Wiggans, T. S. G. Sonstegard, R. D. Schnabel, J. F. Taylor, and F. S. Schenkel. 2009. Invited review: Reliability of genomic predictions for North American Holstein bulls. J. Dairy Sci. 92:16-24. https://doi.org/10.3168/jds .2008-1514.

Vitezica, Z. G., I. Aguilar, I. Misztal, and A. Legarra. 2011. Bias in genomic predictions for populations under selection. Genet. Res. (Camb) 93:357-366. https://doi.org/10.1017/S001667231100022X.

\section{ORCIDS}

I. Granado-Tajada ( https://orcid.org/0000-0002-6557-1467

E. Ugarte $\odot$ https://orcid.org/0000-0002-2231-150X 